\title{
BIOPSIA TRANSRECTAL ECODIRIGIDA DE PRÓSTATA: APORTACIÓN DE UNA UNIDAD UROLÓGICA DE DIAGNÓSTICO ECOGRÁFICO TRAS 10 AÑOS DE EXPERIENCIA.
}

\author{
Rafael Rodríguez-Patrón Rodríguez, Teodoro Mayayo Dehesa, Ricardo García González, \\ Fernándo Arias Fúnez, Alberto Lennie Zuccarino y Enrique Sanz Mayayo.
}

Unidad de Ecografía Urológica. Madrid. España.

\begin{abstract}
Resumen.- OBJETIVO: A pesar de que la Biopsia Transrectal Ecodirigida (BTE) prostática es el método fundamental de diagnóstico del cáncer prostático $(C P)$ no se ha establecido el número ideal de muestras, de repeticiones o la rentabilidad que se le debe exigir. A través de la experiencia de 10 años en BTE pretendemos revisar que puede aportarse a la realización de la prueba y a sus indicaciones.
\end{abstract}

MÉTODO: Se han recogido los datos de PSA, características ecográficas y anatomía patológica de 6000 pacientes a los que se realizó BTE sextante modificada desde 1994 a diciembre de 2002.Para estudiar el papel de la biopsia ampliada se realizo un grupo experimental de 222 pacientes en los que se intentó la obtención de 10 muestras prostáticas mediante BTE. Para determinar la rentabilidad de la biopsia ampliada se estudiará la aportación de las muestras "extras" al rendimiento diagnóstico del grupo experimental (BTE ampliada) y se empleará como grupo control (BTE sextante) y de comparación un conjunto más amplio de 552 pacientes biopsiados en el año 2002. Se estudió la comparabilidad del grupo experimental y control, respecto a las variables recogidas al inicio del estudio.

Rafael Rodríguez-Patrón Rodríguez

Ctra. de Húmera, $55 \mathrm{~F}$

Pozuelo de Alarcón

Madrid. (España)

rafael.rodriguezpatron@wanadoo.es
RESULTADOS: La incidencia de CP en el grupo de 6000 pacientes en la primera biopsia es del 39, $1 \%$ (2345/6000). Los pacientes con PSA entre 4 y 10 $\mathrm{ng} / \mathrm{ml}$ presentan una incidencia mayor del $50 \%$ de cánceres entre las próstatas de menos de 20 cc que disminuye hasta un $8,9 \%$ en la mayores de 50 cc. El porcentaje de CP entre los pacientes con PSA menor de $4 \mathrm{ng} / \mathrm{ml}$ con tacto y ETR normales fue del 16,7\%. La rentabilidad con Densidad de PSA menor de 0,11 ng/ $\mathrm{ml} /$ c.c. fue inferior al 8\%. El PSA L/T muestra una incidencia de CP del 13,7\% con cifras superiores a 0,24. En las regresiones logísticas multivariantes únicamente el PSA L/T no resultó significativo. Entre los pacientes a los que se realizó BTE ampliada 60 (27, 15\%) presentaron CP. Tan sólo el 2,25\% del total de 221 pacientes se beneficiaron del mayor número de muestras. No se han encontrado diferencias significativas en las cifras de CP entre los grupos de biopsia sextante y ampliada. En el análisis de regresión logística multivariante únicamente el PSA y el volumen han resultado significativos, careciendo de influencia en la detección de cáncer el número de muestras, la DPSA y la edad.

CONCLUSIONES: El modelo sextante con obtención de muestras de los cuernos laterales de la próstata sigue siendo la referencia de la BTE mientras que la BTE ampliada no es aplicable de inicio a todos los pacientes por el escaso incremento en la capacidad diagnóstica. EI PSA aislado no puede ser más el referente único para indicar la BTE, siendo, en nuestra experiencia, el volumen un factor decisivo para el ajuste de los niveles de mayor riesgo.

Palabras clave: Biopsia prostática sextante. Biopsia prostática ampliada. Volumen prostático. Ecografía Urológica. 
Summary.- OBJECTIVES: Although transrectal ultrasound-guided (TRUS) prostatic biopsy is the procedure of choice for the diagnosis of prostate cancer (PC), neither the ideal number of cores nor the number of repeated biopsies, nor the required diagnostic yield have been established. After our experience of ten years with TRUS biopsy we perform a review of the technique and its indications.

METHODS: PSA, ultrasound features, and pathologic data of 6000 patients undergoing modified sextant TRUS biopsy between 1994 to December 2002 were collected. 222 patients undergoing ten-core TRUS biopsy were included in an experimental group to study the role of the extended biopsy. The contribution of the extra cores to the diagnostic yield in the experimental group was studied to determine the effectiveness of the extended biopsy, using as a control group 552 patients undergoing sextant TRUS biopsy during 2002. Both groups were comparable for the study variables at the start of the study.

RESULTS: The incidence of PC in the first biopsy in the group of 6000 patients was 39. 1\% (2345/6000). Patients with PSA between 4 and $10 \mathrm{ng} / \mathrm{ml}$ have an incidence of $P C$ greater than 50\% among prostates smaller than $20 \mathrm{cc}$, diminishing down to $8.9 \%$ in those greater than $50 \mathrm{cc}$. The percentage of PC among patients with negative digital rectal examination (DRE), normal TRUS, and PSA below $4 \mathrm{ng} / \mathrm{ml}$ was $16.7 \%$. The diagnostic yield for PSA density lower than 0.11 $\mathrm{ng} / \mathrm{ml} / \mathrm{cc}$ was lower than $8 \%$. The free/total PSA ratio shows a $13.7 \%$ incidence of PC with values higher than 0.24 . Multivariate logistic regression analysis showed that the only non-significant parameter was free/total PSA. Sixty (27.15\%) patients of the extended TRUS biopsy group had PC. Only 2.25\% of the 221 patients benefited from the augmented number of biopsies. There were no significant differences in the figures of prostate cancer between groups. Only PSA and volume where significant in the multivariate logistic regression analysis; number of samples, PSA density and age lacked of influence in the detection of PC.

CONCLUSIONS: The sextant biopsy model obtaining cores from the lateral horns of the prostate continues to be the reference for TRUS biopsy, and the extended biopsy is not applicable to all patients from the beginning do to the small increase in the diagnostic yield. Isolated PSA may not be the unique reference to indicate TRUS biopsy, being volume, in our experience, a definitive factor for the adjustment of high risk levels.

Keywords: Sextant prostatic biopsy. Extended prostatic biopsy. Prostate volume. Urological ultrasound.

\section{INTRODUCCIÓN}

La Biopsia Transrectal Ecodirigida de próstata (BTE) es el elemento básico para el diagnóstico del cáncer prostático (CP). Su inclusión desde hace más de dos décadas supone un bagaje incontestable. A partir de la introducción del PSA apareció una población de pacientes con sospecha de CP por sus cifras elevadas del marcador pero sin evidencia en el tacto rectal (TR) - la ETR, lo que hacía necesaria la sistematización de la biopsia en aras de optimizar sus resultados. Hodge y cols (1) fueron los primeros en proponer la realización de estas biopsias de forma reglada, obteniendo tres muestras de cada lóbulo prostático a mitad de camino entre el margen externo de la glándula y la línea media, formando un ángulo aproximado de 45 . Este modelo ha constituido la base de la biopsia para muchos grupos en el mundo y aunque se han propuesto modificaciones posteriores siempre ha constituido la referencia obligada para comparar resultados. La efectividad obteniendo 6 muestras es elevada aunque a pesar de ello la sensibilidad de la BTE se sitúa en torno al $70-80 \%(2,3)$. Esto ha hecho que en los últimos años se plantee la necesidad de obtener mayor número de muestras para paliar la falta de sensibilidad. A pesar de ello no queda claro cual es el número ideal de cilindros para alcanzar el equilibrio entre efectividad, tolerancia, efectos adversos y evitar el diagnóstico de un número excesivo de los llamados tumores "poco significativos". Tampoco se ha establecido de forma definitiva cuantas biopsias se pueden llegar a hacer para tener una seguridad suficiente de que no pasamos por alto un tumor, $\mathrm{o}$ la necesidad o no de anestesia para la realización del procedimiento.

Desde el inicio en nuestra Unidad de la realización de BTE se optó por la obtención sextante, modificando la técnica de Hodge y cols (1) hacia los aspectos más laterales de la zona periférica de forma que el cilindro obtenido procediera puramente de esta sin incluir en la medida de lo posible zona transicional que inevitablemente aparece en los cilindros obtenidos según la BTE clásica. A continuación pretendemos repasar la experiencia de estos años y que aportación podemos realizar a la realización de la BTE y a sus indicaciones.

\section{MATERIAL Y MÉTODO}

Se han recogido los datos de PSA, características ecográficas y anatomía patológica de 6000 pacientes a los que se realizó BTE sextante modificada desde 1994 a diciembre de 2002. De los 6000 pacientes 674 han sido biopsiados 2 veces, 128 tres veces, 43 cuatro veces, 13 en cinco ocasiones, 2 en seis y 1 a un paciente se le practicaron 7 biopsias. En conjunto suman 7127 biopsias. 
Para estudiar el papel de la biopsia ampliada se realizo un grupo experimental de 222 pacientes en los que se intentó la obtención de 10 muestras prostáticas mediante BTE. Después de realizar las primeras 50 se añadió anestesia local al procedimiento al observar la mala tolerancia al procedimiento sin ella. Los pacientes de este grupo debían ser menores de 75 años, tener TR y ETR normales, ser primeras biopsias y no tener patología que incrementase el riesgo de la BTE o sus complicaciones. A los pacientes se les entregó un cuestionario de tolerancia y se recogieron telefónicamente las complicaciones posteriores.

De las 10 muestras, 6 se obtuvieron mediante BTE sextante modificada según nuestra técnica habitual (4). Para completar las diez previstas se añadieron 2 muestras "extras" de la región apical-dorsal de la zona periférica de cada lado que se enviaron en los primeros 50 pacientes en un único envase y en los restantes en un bote para cada lóbulo prostático. La zona de obtención de estas muestras "extras" se decidió tomando en cuenta el estudio de Mazaal y cols (5) en el que se determinó la localización de los tumores que no habían sido diagnosticados en una primera biopsia sextante y que observó una concentración de los mismos en la región apical y posterior de la zona periférica.

Las exploraciones se han realizado con ecógrafos de la línea Sonoline de Siemens Medical Systems, Inc. Ultrasound Group, Issaquah, WA. Para la obtención de muestras se utilizan agujas 18Gauge tipo Tru-Cut modificadas para ser disparadas mediante el dispositivo Biopty-Bard $®$ (Bard urological Inc., Atlanta, Georgia), que obtienen cilindros de tejido de $1,5-2 \mathrm{~cm}$ de longitud con un diámetro de aproximadamente $1 \mathrm{~mm}$.

La preparación previa incluyó la aplicación de un enema comercial, ciprofloxacino o levofloxacino y metamizol magnésico dos horas antes de la prueba.

Para determinar la rentabilidad de la biopsia ampliada se estudiará la aportación de las muestras "extras" al rendimiento diagnóstico del grupo experimental (BTE ampliada) y se empleará como grupo control (BTE sextante)y de comparación un conjunto más amplio de 552 pacientes biopsiados en el año 2002 seleccionados entre los 6000 recogidos de BTE sextante modificada entre 1994 y 2002, tomando aquellos con criterios similares (ausencia de sospecha por TR o ETR, primera biopsia). Se estudió la comparabilidad del grupo experimental y control, respecto a las variables recogidas al inicio del estudio. Para ello se utilizarán test estadísticos de comparación de medias (t de Student) y de porcentajes ( $\chi^{2}$ cuadrado, test de Fisher), y en algún caso de ausencia de normalizada, pruebas no paramétricas.

El análisis bivariante y multivariante se realiza por ajuste a protocolo. Se comparó el porcentaje de detección en cada grupo y en el grupo de 10 cilindros se utilizó el test de la Chi cuadrado de McNemar para estudiar si había diferencias en el porcentaje de detección con las muestras adicionales de este grupo. Se calcularon intervalos de confianza al $95 \%$ respecto a los variables del estudio en cada grupo. Para algunas variables cuantitativas continuas, se realizó análisis de la varianza para estudiar la relación de estas variables según grupos y según otras variables predictoras relacionadas.

TABLA I. INCIDENCIA DE ANATOMIIA PATOLÓGICA EN SUCESIVAS BIOPSIAS.

\begin{tabular}{|c|c|c|c|c|c|}
\hline \multicolumn{7}{|c|}{$\mathbf{N}(\%)$} \\
\hline$N^{\circ}$ biopsia & $\mathbf{N}$ & HPB (\%) & CÁNCER(\%) & PIN(\%) & AGF(\%) \\
\hline $1^{\underline{a}}$ & 6000 & $3406(56,8)$ & $2345(39,1)$ & $122(2)$ & $127(2,1)$ \\
\hline $2^{\underline{a}}$ & 832 & $611(73,4)$ & $168(20,2)$ & $25(3)$ & $28(3,4)$ \\
\hline $3^{\underline{a}}$ & 185 & $143(77,3 \%)$ & $31(16,8)$ & $5(2,7)$ & $6(3,2)$ \\
\hline $4^{\underline{a}}$ & 59 & $46(78)$ & $7(11,9)$ & $2(3,4)$ & $4(6,8)$ \\
\hline $5^{\underline{a}}$ & 16 & $11(68,8 \%)$ & $3(18,8)$ & $1(6,3)$ & $1(6,3)$ \\
\hline $6^{\underline{a}}$ & 3 & $2(66,7)$ & & $1(33,3)$ & \\
\hline $7^{\underline{a}}$ & 1 & & & $1(100)$ & \\
\hline
\end{tabular}


El análisis se ha realizado con el paquete estadístico SPSS 11.0 y los análisis multivariantes con SAS software release 8.2. Los datos fueron introducidos en una base de datos SPSS.

\section{RESULTADOS}

\section{BIOPSIA SEXTANTE}

La incidencia de CP en el grupo de 6000 pacientes en la primera biopsia es del $39,1 \%$ (2345/6000). En las sucesivas biopsias el porcentaje de CP diagnosticados va disminuyendo no apareciendo tumores entre los pacientes con sexta o séptima biopsia (Tabla I). La incidencia conjunta de CP en la población biopsiada fue del 42,6\% (2554/6000). Las biopsias sucesivas incrementan el diagnostico de $\mathrm{CP}$ en un $8,2 \%$ entre los pacientes con cáncer y un $3,5 \%$ sobre el total de pacientes biopsiados. El porcentaje de pacientes con un tumor moderadamente o bien diferenciado (Gleason igual o menor a 6) se incrementa en las sucesivas biopsias desde el $41,8 \%$ en la primera hasta un $70 \%$ en la tercera.

En la primera BTE cuando el tacto fue sospechoso la biopsia encontró un $82,6 \%$ de CP. La existencia de nódulos hipoecoicos o próstata heterogénea se asoció a un 78,2 y $82,9 \%$ de tumores respectivamente. Tanto en el caso de la ecografía como el TR la asociación con la patología resultó significativa mediante el test de Cochran-Mantel-Haenzel con $\mathrm{p}<0,0001$.

Se han establecido rangos de volumen prostático para estudiar la incidencia de cáncer en ellos. Estos rangos son los siguientes: próstatas menores de $20 \mathrm{cc}$, de 20 a $30 \mathrm{cc}$, de 30 a $40 \mathrm{cc}$, de 40 a 50 cc y mayores de $50 \mathrm{cc}$ la tabla 2 recoge la incidencia de la anatomía patológica en los distintos grupos de volumen en la primera biopsia, en total y en aquellos pacientes con PSA entre 4 y $10 \mathrm{ng} / \mathrm{ml}$, y en segunda biopsia. Se observa una alta incidencia de CP en

TABLA II. INCIDENCIA DE ANATOMÍA PATOLÓGICA SEGÚN RANGOS DE VOLUMEN.

\begin{tabular}{|c|c|c|c|c|c|c|}
\hline \multicolumn{7}{|c|}{$\mathbf{N}(\%)$} \\
\hline $\begin{array}{c}\text { Grupos } \\
\text { volumen }(c, c) \text {, }\end{array}$ & & $\mathbf{n}$ & HBP & CANCER & PIN & AGF \\
\hline \multirow[t]{3}{*}{$<20$} & $1 B$ & 875 & $259(29,6)$ & $588(67,2)$ & $10(1,1)$ & $18(2,1)$ \\
\hline & $1 B^{*}$ & 251 & $111(44,2)$ & $131(52,2)$ & $4(1,6)$ & $5(2,0)$ \\
\hline & $2 B$ & 48 & $18(37,5)$ & $26(54,2)$ & $2(4,2)$ & $2(4,2)$ \\
\hline \multirow[t]{3}{*}{$20-30$} & $1 B$ & 1468 & $665(45,3)$ & $743(50,6)$ & $24(1,6)$ & $36(2,5)$ \\
\hline & $1 B^{*}$ & 612 & $414(67,6)$ & $161(26,3)$ & $17(2,8)$ & $20(3,3)$ \\
\hline & $2 B$ & 139 & $78(56,1)$ & $48(34,5)$ & $5(3,6)$ & $8(5,8)$ \\
\hline \multirow[t]{3}{*}{$30-40$} & $1 B$ & 1397 & $787(60,2)$ & $465(35,6)$ & $28(2,1)$ & $27(2,1)$ \\
\hline & $1 B^{*}$ & 612 & $414(67,6)$ & $161(26,3)$ & $17(2,8)$ & $20(3,3)$ \\
\hline & $2 B$ & 174 & $122(70,1)$ & $36(20,7)$ & $6(3,4)$ & $10(5,7)$ \\
\hline \multirow[t]{3}{*}{$40-50$} & $1 B$ & 858 & $565(65,9)$ & $251(29,3)$ & $24(2,8)$ & $18(2,1)$ \\
\hline & $1 B^{*}$ & 431 & $352(81,7)$ & $55(12,8)$ & $17(3,9)$ & $7(1,6)$ \\
\hline & $2 B$ & 146 & $114(78,1)$ & $23(15,8)$ & $5(3,4)$ & $4(2,7)$ \\
\hline \multirow[t]{3}{*}{$>50$} & $1 \mathrm{~B}$ & 1464 & $1112(76)$ & $289(19,7)$ & $36(2,5)$ & $27(1,8)$ \\
\hline & $1 B^{*}$ & 653 & $568(87,0)$ & $58(8,9)$ & $14(2,1)$ & $13(2,0)$ \\
\hline & $2 B$ & 315 & $271(86,0)$ & $34(10,8)$ & $6(1,9)$ & $4(1,3)$ \\
\hline
\end{tabular}

$1 B=$ primera biopsia; $1 B^{*}=$ primera biopsia en pacientes con PSA entre 4 y $10 \mathrm{ng} / \mathrm{ml}$; $2 B=$ segunda biopsia 
TABLA III. INCIDENCIA DE LAS DISTINTAS ANATOMIAS PATOLÓGICAS SEGÚN RANGOS DE PSA.

\begin{tabular}{|c|c|c|c|c|c|}
\hline \multicolumn{5}{|c|}{ N(\%) } & \\
\hline Rangos & HPB (\%) & CÁNCER(\%) & PIN(\%) & AGF(\%) & TOTAL \\
\hline PSA(ng/ml) & & & & & \\
\hline$<4$ & $144(64,3)$ & $68(30,4)$ & $2(0,9)$ & $10(4,5)$ & 224 \\
\hline $4-10$ & $2274(66,1)$ & $1017(29,6)$ & $78(2,3)$ & $71(2,1)$ & 3440 \\
\hline $10-20$ & $726(51,7)$ & $618(44)$ & $32(2,3)$ & $29(2,1)$ & 1405 \\
\hline $20-30$ & $130(40,5)$ & $177(55,1)$ & $6(1,9)$ & $8(2,5)$ & 321 \\
\hline $30-50$ & $53(25,1)$ & $153(72,5)$ & $1(0,5)$ & $4(1,9)$ & 211 \\
\hline$>50$ & $16(5,4)$ & $274(92,9)$ & $2(0,7)$ & $3(1)$ & 295 \\
\hline
\end{tabular}

las próstatas pequeñas que alcanza el $67,2 \%$ en las de tamaño normal (menores de $20 \mathrm{cc}$ ) que disminuye a medida que aumenta el volumen encontrando únicamente un $19,7 \%$ en aquellas mayores de $50 \mathrm{cc}$ $(p<0,0001)$. En los pacientes con PSA entre 4 y 10 $\mathrm{ng} / \mathrm{ml}$ se aprecia similar relación con una incidencia mayor del $50 \%$ de cánceres entre las próstatas de menos de $20 \mathrm{cc}$ que disminuye con el incremento de volumen hasta resultar de un $8,9 \%$ en la mayores de 50 cc y $10,4 \%$ en las mayores de 40 cc. En la segun- da biopsia, los resultados son muy similares a los de pacientes con PSA entre 4 y $10 \mathrm{ng} / \mathrm{ml}$ en la primera biopsia (Tabla II).

Se han establecido rangos de PSA para estudiar la incidencia de CP en ellos (Tabla III). El porcentaje entre los pacientes con PSA menor de 4ng/ $\mathrm{ml}(\mathrm{n}=224)$ fue del $30,4 \%$ aunque considerando los 108 pacientes con tacto y ETR normales la incidencia fue del $16,7 \%$ (18 pacientes). La mediana de volu-

TABLA IV. INCIDENCIA DE CÁNCER SEGÚN RANGOS DE DPSA Y PSA L/T EN PRIMERA BIOPSIA.

\begin{tabular}{|c|c|c|c|c|c|}
\hline $\begin{array}{c}\text { Rango DPSA } \\
\text { (ng/ml/c.c.) }\end{array}$ & $\mathbf{n}$ & $\begin{array}{c}\text { Cáncer } \\
\mathbf{n}(\%)\end{array}$ & $\begin{array}{c}\text { RangoPSA } \\
\text { L/T }\end{array}$ & $\mathbf{n}$ & $\begin{array}{c}\text { Cáncer } \\
\mathbf{n}(\%)\end{array}$ \\
\hline$<0.11$ & 456 & $36(7.9)$ & $>0.24$ & 211 & $29(13.7)$ \\
\hline $0.11-0.14$ & 458 & $46(10)$ & $0-19-0.24$ & 238 & $41(17.2)$ \\
\hline $0.14-0.17$ & 534 & $72(13.5)$ & $0.17-0.19$ & 150 & $23(15.3)$ \\
\hline $0.17-0.20$ & 447 & $67(15)$ & $0.15-0.17$ & 189 & $32(16.9)$ \\
\hline $0.20-0.23$ & 381 & $85(22.3)$ & $0.13-0.15$ & 238 & $50(21)$ \\
\hline $0.23-0.29$ & 489 & $125(25.6)$ & $0.11-0.13$ & 216 & $55(25.5)$ \\
\hline $0.29-0.37$ & 379 & $115(30.3)$ & $0.10-0.11$ & 93 & $34(36.6)$ \\
\hline $0.37-0.52$ & 339 & $131(38.6)$ & $0.08-0.10$ & 165 & $50(30.3)$ \\
\hline $0.52-0.92$ & 236 & $105(44.5)$ & $0.06-0.08$ & 117 & $52(44.4)$ \\
\hline$>0.92$ & 109 & $58(53.2)$ & $<0.06$ & 85 & $33(38.8)$ \\
\hline total & $\mathbf{3 8 2 8}$ & $\mathbf{8 4 0 ( 2 1 . 9 )}$ & total & $\mathbf{1 7 0 2}$ & $\mathbf{3 9 9 ( 2 3 . 4 )}$ \\
\hline
\end{tabular}


men en ellos se situó en 24,2 c.c. siendo el $95 \%$ de ellas menores de 40 c.c.

Para el total de primeras biopsias (Tabla IV) los rangos establecidos de DPSA muestran una rentabilidad por debajo del $8 \%$ con densidades menores de $0,11 \mathrm{ng} / \mathrm{ml} /$ c.c. y alrededor del $10 \%$ si se estableciera el límite en 0,14. Datos similares se obtuvieron entre pacientes con PSA intermedio. EI PSA L/T es menos discriminativo con los rangos obtenidos encontrando una incidencia de CP del $13,7 \%$ con cifras superiores a 0,24 que es el grupo superior (Tabla IV). En pacientes con PSA entre 4 y $10 \mathrm{ng} / \mathrm{ml}$ este mismo grupo presenta una incidencia de $12,9 \%$.

En las regresiones logísticas multivariantes que incluyen PSA, DPSA, volumen fraccionados en categorías, el TR y la presencia de una ecografía sospechosa (nódulos hipoecoicos o próstata heterogénea) todos los factores resultaron significativos (Tabla V). La combinación de pruebas consigue un área bajo la curva ROC de 0,84. El PSA L/T no resultó significativo en un estudio de regresión aparte con los pacientes en los que se disponía de este dato y que incorporaba además las mismas categorías de DPSA y volumen, TR y ecografía. El PSA L/T.

\section{BIOPSIA AMPLIADA}

Entre los pacientes a los que se realizó BTE ampliada $146(66,06 \%)$ mostraban HBP, $60(27,15 \%)$ CP, 5 (2,26\%) PIN y 10 (4,52\%) AGF. En un paciente no se obtuvieron muestras al no tolerar el paso del transductor. Los datos de edad, PSA, DPSA, PSA L/T y volumen se muestran en la Tabla VI.

Tan sólo 5 de los 60 pacientes con cáncer $(8,33 \%)$ fueron diagnosticados en base a los cilindros extras de modo que sólo el $2,25 \%$ del total de 221 pacientes $(5 / 221)$ se beneficiaron del mayor número de muestras. El test de $\mathrm{McNemar}$ señala que las muestras laterales detectan $\mathrm{CP}$ con mayor frecuencia que las muestras extras $(p<0,001)$. Al igual que en el grupo total de biopsia sextante se ha calculado la incidencia de $\mathrm{CP}$ en función de los mismos rangos de volumen empleados anteriormente, encontrando una distribución similar con un $69,2 \%$ en las próstatas menores o iguales a 20c.c, que disminuye a medida que aumenta el tamaño hasta una incidencia del $11,6 \%$ en glándulas mayores de 50 c.c. $(p<0,0001$ en el test de tendencia de Cochran-Armitage).

\section{COMPARACIÓN BIOPSIA AMPLIADA VS SEXTANTE}

La Tabla VII muestra los descriptivos de ambos grupos para el PSA y volumen.

TABLA V. REGRESIÓN MULTIVARIANTE PARA PSA, DPSA, VOLUMEN, TR Y ETR.

\begin{tabular}{|c|c|c|c|c|c|}
\hline Parametro & \multirow{2}{*}{$\chi^{2}$ Wald } & \multirow{2}{*}{ Pr $>\chi^{2}$} & \multicolumn{3}{|c|}{ Odds ratio estimado } \\
\cline { 4 - 6 } & & & Punto estimado & \multicolumn{2}{c|}{ Intervalo confianza al 95\% } \\
\hline PSA 6 -10 & 0.1575 & 0.6915 & 1.042 & 0.850 & 1.278 \\
\hline PSA 10-33 & 4.7555 & 0.0292 & 1.350 & 1.031 & 1.768 \\
\hline PSA >33 & 39.4028 & $<.0001$ & 3.915 & 2.556 & 5.994 \\
\hline DPSA 0.13-0.18 & 2.2377 & 0.1347 & 1.245 & 0.934 & 1.659 \\
\hline DPSA 0.18-0.26 & 14.4893 & 0.0001 & 1.758 & 1.315 & 2.351 \\
\hline DPSA $\geq 0.26$ & 42.9228 & $<.0001$ & 3.124 & 2.222 & 4.392 \\
\hline Volumen 20-30 & 11.4498 & 0.0007 & 0.692 & 0.559 & 0.856 \\
\hline Volumen 30-40 & 37.0644 & $<.0001$ & 0.470 & 0.368 & 0.599 \\
\hline Volumen 40-50 & 40.9086 & $<.0001$ & 0.391 & 0.293 & 0.521 \\
\hline Volumen >50 & 73.1645 & $<.0001$ & 0.256 & 0.188 & 0.350 \\
\hline TR sospechoso & 170.9448 & $<.0001$ & 3.743 & 3.071 & 4.563 \\
\hline ETR sospechosa & 228.8563 & $<.0001$ & 3.535 & 3.001 & 4.163 \\
\hline
\end{tabular}

Likelihood Ratio: $\chi^{2} 2263.67 \mathrm{GL}=12$ p<0, 000 1.PSA(ng/ml); DPSA (ng/ml/c.c.); Volumen (c.c.) 
TABLA VI. DATOS ANALÍTICOS, VOLUMEN PROSTÁTICO Y EDAD EN LOS PACIENTES BIOPSIADOS.

\begin{tabular}{|c|c|c|c|c|c|}
\hline & No pacientes & rango & media & DT & Mediana \\
\hline Edad (años) & 214 & $42-75$ & 63,23 & 7,15 & 64 \\
\hline Vol.prostático (c.c.) & 220 & $11-122$ & 39,25 & 19,72 & 35 \\
\hline$P S A(n g / m l)$ & 215 & $4,01-33,8$ & 7,45 & 3,96 & 6,36 \\
\hline DPSA(ng/ml/c.c.) & 214 & $0,06-1,12$ & 0,22 & 0,13 & 0,18 \\
\hline PSA L/T & 56 & $0,02-0,31$ & 0,15 & 0,06 & 0,14 \\
\hline
\end{tabular}

El grupo de BTE sextante mostraba una incidencia de CP del 24,5\% (135/552).

El grupo de BTE ampliada presenta un $27,15 \%$ de CP y un $24,9 \%$ sin contar con la aportación de las muestras extras. No se han encontrado diferencias significativas en las cifras de CP incluidas las muestras extras.

En el análisis de regresión logística multivariante únicamente el PSA y el volumen han resultado significativos, careciendo de influencia en la detección de cáncer el número de muestras y la DPSA (Tabla VIII).

La edad tampoco ha mostrado relación significativa con la probabilidad de $C P$, considerado únicamente en el grupo de biopsia ampliada dado que el de comparación no se disponía de este dato de forma regular.

\section{DISCUSIÓN}

Desde el inicio de la comunicación de los resultados de nuestro grupo se ha constatado una reducción de la incidencia de $\mathrm{CP}$ en la BTE con el tiempo, desde un $45 \%(6,7)$ a un $40 \%(4)$ y el $39 \%$ actual que continúa siendo una cifra muy elevada para la BTE sextante, si bien la toma de muestras de los cuernos laterales prostáticos presenta una rentabilidad mayor que la BTE clásica descrita por Hodge y cols (1). Esto hace posiblemente que frente a las series americanas que toman como base este modelo la incidencia de esta serie se aproxime más a la de trabajos de biopsia ampliada (8-15), en los que únicamente Presti y cols presentan cifras escasamente superiores al $40 \%(8,15)$.

Aunque algunos autores no tienen en consideración el aspecto ecográfico o no han encontrado relación entre este y la presencia de CP (16-18) la

TABLA VII. CARATERÍSTICAS DE PSA Y VOLUMEN EN LOS GRUPOS COMPARADOS.

\begin{tabular}{|c|c|c|c|c|}
\hline & \multicolumn{2}{|c|}{ Grupo BTE sextante(n=551) } & \multicolumn{2}{c|}{ Grupo BTE ampliada(n=220) } \\
\hline & PSA(ng/ml) & VOLUMEN(c.c.) & PSA(ng/ml) & VOLUMEN(c.c.) \\
\hline $\mathrm{N}$ & 537 & 551 & 215 & 220 \\
\hline Mediana & 6,71 & 35,59 & 6,36 & 35,00 \\
\hline Media & 7,97 & 41,37 & 7,45 & 39,25 \\
\hline DT & 5,00 & 22,85 & 3,96 & 19,72 \\
\hline Varianza & 25,04 & 522,41 & 15,73 & 388,92 \\
\hline Rango & 46,00 & 209 & 29,79 & 111,00 \\
\hline
\end{tabular}

La prueba t no encontró diferencias significativas entre ambos en las cifras de PSA, volumen o DPSA. 
especificidad del TR y de la ETR continúa siendo muy elevada, por lo que a pesar de que la incidencia de anomalías en estas exploraciones puede haberse reducido con el tiempo no debe olvidarse su importancia a la hora de planificar la BTE.

Es llamativo el descenso de biopsias positivas a medida que se incrementa el volumen llegando a ser inferior al $9 \%$ en próstatas mayores de $50 \mathrm{cc}$ con PSA entre 4 y $10 \mathrm{ng} / \mathrm{ml}$. La primera justificación a esto sería pensar que el número de muestras es insuficiente en próstatas de mayor tamaño. Aunque igual de inmediato es pensar que, como se sabe desde hace tiempo, a mayor adenoma prostático mayores cifras de PSA. Al igual que Remzi y cols (19) y Diavan y cols (20) se encuentra un mayor volumen de las glándulas con tumor que se diagnosticaron en la segunda biopsia frente a las que se diagnosticaron en la primera, si bien la mediana no supera en biopsias sucesivas los $35 \mathrm{cc}$ lo que parece indicar que en próstatas de mayor tamaño continúa sin diagnosticarse tumor a pesar de la repetición de biopsias con el incremento de muestras consiguiente. En pacientes con PSA entre 4 y $10 \mathrm{ng} / \mathrm{ml}$ en la segunda BTE el $70 \%$ de CP tuvieron un volumen inferior a $40 \mathrm{cc}$. Otros autores han referido una relación similar entre volumen y frecuencia de CP (21-23). Esto concuerda con los datos encontrados respecto a la DPSA como parece lógico puesto que se trata de un parámetro directamente ligado al volumen prostático. Así se encontró menos de un $8 \%$ de CP en la primera biopsia cuando la DPSA fue inferior al $0,11 \mathrm{ng} / \mathrm{ml} / \mathrm{cc}$ y alrededor del 10\% con un límite de 0,14. Apoyando los datos encontrados respecto al volumen la relación entre este y la frecuencia de CP es similar en pacientes con biopsia ampliada a 10 cilindros donde por encima de $50 \mathrm{cc}$ sólo el 11,6\% presentaban tumor independientemente de las cifras de PSA. Además considerando los pacientes que se diagnosticaron en base a las muestras extras, sólo uno de ellos presentaba un volumen superior a $35 \mathrm{cc}$ lo que no apoya la idea de que este tipo de biopsia sea más rentable en próstatas de mayor volumen.

Todos los datos anteriores nos hacen pensar que la menor incidencia de $\mathrm{CP}$ en próstatas de mayor volumen no se debe al menor número de muestras sino a la menor incidencia para unas cifras dadas de PSA y que la elevación de este es principalmente debido al componente de HPB, al igual que es la HPB la que condiciona el aumento de la glándula permaneciendo la zona periférica, asiento fundamental del cáncer, con un volumen similar. Esto no significa que no vayan a aparecer tumores en próstatas de mayor volumen pero en ausencia de alteraciones en el TR o en la ETR y con PSA intermedio la incidencia será baja y previsiblemente puedan ser de características más favorables aunque este punto no podamos confirmarlo en ausencia de datos de prostatectomías radicales, aunque Catalona y cols (24) refieren que en pacientes diagnosticados de CP con DPSA menor de $0,15 \mathrm{ng} / \mathrm{ml} /$ c.c. la probabilidad de presentar un tumor de características favorables en la pieza de prostatectomía (tumor organoconfinado, Gleason menor de 7 y menos del $10 \%$ de afectación) es del $75 \%$ frente a sólo un tercio cuando este índice es mayor de 0,15. Stamey en el comentario editorial a un artículo de Eskew y cols (16) dice que, en determinado sentido, el volumen podría considerarse como un marcador tumoral similar al PSA puesto que cuanto mayor es una próstata mayor es la probabilidad de curación con prostatectomía radical.

TABLA VIII. REGRESIÓN LOGÍSTICA EN EL GRUPO CONJUNTO COMPARADO. NI EL GRUPO (6 VS 10 CILINDROS), NI LA DPSA FUERON SIGNIFICATIVAS.

\begin{tabular}{|c|c|c|c|c|c|}
\hline \multirow{2}{*}{ *parametro } & \multirow{2}{*}{$\chi^{2}$ Wald } & \multirow{2}{*}{$\operatorname{Pr}>\chi^{2}$} & \multicolumn{3}{|c|}{ Odds ratio estimado } \\
\cline { 4 - 6 } & & & Punto estimado & Intervalo confianza al 95\% \\
\hline PSA TOTAL & 26,79 & $<0,0001$ & 1,11 & 1,068 & 1,156 \\
\hline Volumen 20-30 vs $<20$ & 17,03 & $<0,0001$ & 0,30 & 0,17 & 0,53 \\
\hline Volumen 30-40 vs <20 & 37,29 & $<0,0001$ & 0,16 & 0,088 & 0,288 \\
\hline Volumen 40-50 vs <20 & 43,37 & $<0,0001$ & 0,08 & 0,037 & 0,167 \\
\hline Volumen $>50$ vs $<20$ & 69,29 & $<0,0001$ & 0,05 & 0,024 & 0,098 \\
\hline
\end{tabular}

Likelihood Ratio: $\chi^{2}=114,07 \mathrm{GL}=5 \mathrm{p}<0,001$. Para cada grupo de volumen se calcula el Odds Ratio frente a tener un volumen menor de 20 c.c. 
Nos ha resultado llamativo que en los análisis multivariantes todos los factores estudiados hayan sido significativos a excepción de la relación PSA L/T que sí lo fue en el análisis univariante.

Parece lógico que a mayor número de muestras mayor sea el diagnóstico de CP pero la incidencia no fue significativamente diferente entre los grupos comparados de BTE sextante y ampliada aunque fue mayor en estos últimos. Sólo el 2,25\% del total de pacientes se beneficiaron del mayor número de muestras. En el análisis multivariante ni el número de muestras ni la edad se relacionaron con el diagnóstico de CP.

En pacientes con TR normal la frecuencia de tumor registrada por otros autores en BTE ampliada oscila entre el 28 y el $34,8 \%(8,11-14,16)$. Las cifras encontradas en esta serie del $27,2 \%$ son algo inferiores aunque no es posible conocer cuantos de los pacientes referidos presentaban una ecografía sospechosa lo que sin duda incrementaría el porcentaje de biopsias positivas.

Este incremento diagnóstico fue a expensas de un aumento de los efectos secundarios sobre todo de rectorragia que se situaba en torno al $4 \%$ y la necesidad del empleo de anestesia local debido a la peor tolerancia frente a la BTE sextante (25).

Thompson y cols (26) encuentran un 15,2\% de CP en pacientes con PSA inferior a $4 \mathrm{ng} / \mathrm{ml}$ comparable al $16,7 \%$ de esta serie en pacientes con ETR y TR normales. Cuando fue menor de $0,5 \mathrm{ng} / \mathrm{ml}$ la incidencia era del $6,6 \%, 10,1 \%$ con PSA entre 0,6 y $1 \mathrm{ng} / \mathrm{ml}, 17 \%$ con PSA entre 1,1 y $2 \mathrm{ng} / \mathrm{ml}, 23,9 \%$ con PSA entre 2,1 y $3 \mathrm{ng} / \mathrm{ml}$ y $26,9 \%$ con PSA entre 3,1 y $4 \mathrm{ng} / \mathrm{ml}$. La mayoría de estos pacientes fueron sometidos a BTE sextante. Si comparamos estas cifras con lo encontrado en este trabajo observamos similar rentabilidad en la biopsia entre pacientes con volumen superior a $50 \mathrm{cc}$ y PSA entre 4 y $10 \mathrm{ng} / \mathrm{ml}$ o DPSA inferior a $0,14 \mathrm{ng} / \mathrm{ml} / \mathrm{cc}$ que en aquellos con PSA inferior a $1 \mathrm{ng} / \mathrm{ml}$ del trabajo de Thompson y cols. Si pensamos que estos datos son extrapolables a nuestro medio quedan dos posturas posibles ante la indicación de la BTE: se abandona la realización del PSA y se biopsia a todo varón mayor de 50 años que quiera saber si puede tener un $\mathrm{CP}$ o se acota un grupo de mayor riesgo asumiendo que en el proceso se dejarán de diagnosticar algunos cánceres. Esta posición parece hoy por hoy más razonable hasta la existencia de mayor evidencia sobre la utilidad del diagnóstico precoz en el CP y conllevaría según los datos obtenidos a recortar las indicaciones en pacientes con próstatas de mayor volumen, PSA intermedio y ausencia de anomalías en el TR o la ETR, y a incrementarlas en pacientes con PSA inferior a $4 \mathrm{ng} / \mathrm{ml}$ y glándulas de tamaño menor.

\section{CONCLUSIONES}

Tras 10 años de experiencia y con los datos recogidos pensamos que una buena ETR continúa siendo un aspecto fundamental en la BTE incrementando su rentabilidad. El modelo sextante con obtención de muestras de los cuernos laterales de la próstata sigue siendo la referencia de la BTE mientras que la BTE ampliada no es aplicable de inicio a todos los pacientes por el escaso incremento en la capacidad diagnóstica. El PSA aislado no puede ser más el referente único para indicar la BTE, siendo, en nuestra experiencia, el volumen un factor decisivo para el ajuste de los niveles de mayor riesgo.

\section{BIBLIOGRAFIA y LECTURAS RECOMENDADAS (*lectura de interés $y^{* *}$ lectura fundamental)}

1. HODGE, K.K.; McNEAL, J.E.; TERRIS, M.K. y cols.: "Random systematic versus directed ultrasound guided transrectal core biopsies of the prostate". J. Urol., 142: 71, 1989.

*2. RABBANI, F.; STROUMBAKIS, N.; KAVA, B.R. y cols.: "Incidence and clinical significance of false negative sextant prostate biopsies". J. Urol., 159: 1247, 1998.

*3. TERRIS, M.K.: "Sensitivity and specificity of sextant biopsies in the detection of prostate cancer: preliminary report". Urology, 54: 486, 1999.

*4. MAYAYO, T.; RODRÍGUEZ-PATRÓN, R.; LENNIE, A. y cols.: "Biopsia transrectal ecodirigida. Análisis de los resultados de una serie de 1900 pacientes". Arch. Esp. Urol., 52: 453, 1999.

5. MAZAL, P.R.; HAITEL, A.; WINDISCHBERGER, C. y cols.: "Spatial distribution of prostate cancers undetected on initial needle biopsies". Eur. Urol., 39: 662, 2001.

6. RODRÍGUEZ, R.; MAYAYO, T.; GALBIS, F. y cols.: "Utilidad clínica de las pruebas diagnósticas disponibles en el carcinoma prostático. Resultados sobre 500 biopsias. Parte II: tacto rectal, PSA y ecografía transrectal”. Arch. Esp. Urol., 50: 339, 1997.

7. RODRÍGUEZ, R.; MAYAYO, T.; GALBIS, F. y cols.: "Inflamación prostática crónica: un factor de confusión en el diagnóstico del cáncer prostático". Arch. Esp. Urol., 49: 669, 1996.

**8. PRESTI, J.C. Jr.; O’DOWD, G.J.; MILLER, M.C. y cols.: "Extended peripheral zone biopsy schemes increase cancer detection rates and mini- 
mize variance in prostate specific antigen and age related cancer rates: results of a community multipractice study". J. Urol., 169: 125, 2003.

9. RODRÍGUEZ DUARTE, C.: "Biopsia prostática multifragmentaria". Arch. Esp. Urol., 55: 907, 2002.

*10. BABAIAN, R.J.; TOI, A.; KAMOI, K. y cols.: "Comparative analysis of sextant and an extended 11-core multisite directed biopsy strategy". J. Urol., 163: 152, 2000.

*11. LEVINE, M.A.; ITTMAN, M.; MELAMED, J. y cols.: "Two consecutive sets of transrectal ultrasound guided sextant biopsies of the prostate for the detection of prostate cancer". J. Urol., 159: 471, 1998.

*12. DE LA TAILlE, A.; ANTIPHON, P.; SALOMON, L. y cols.: "Prospective evaluation of a 21-sample needle biopsy procedure designed to improve the prostate cancer detection rate". Urology, 61: 1181, 2003.

*13. RAVERY, V.; GOLDBLATT, L.; ROYER, B. y cols.: "Extensive biopsy protocol improves the detection rate of prostate cancer". J. Urol., 164: 393,2000

*14. NAUGHTON, C.K.; MILLER, D.C.; MAGER, D.E. y cols.: "A prospective randomized trial comparing 6 versus 12 prostate biopsy cores: impact on cancer detection". J. Urol., 164: 388, 2000.

*15. PRESTI, J.C. Jr.; CHANG, J.J.; BHARGAVA, V. y cols.: "The optimal systematic prostate biopsy scheme should include 8 rather than 6 biopsies: results of a prospective clinical trial". J. Urol., 163: 163, 2000.

*16. ESKEW, L.A.; BARE, R.L.; McCULLOUGH, D.L.: "Systematic 5 region prostate biopsy is superior to sextant method for diagnosing carcinoma of the prostate". J. Urol., 157: 199, 1997.

17. APPLEWHITE, J.C.; MATLAGA, B.R.; McCULLOUGH, D.L.: "Results of the 5 region prostate biopsy method: the repeat biopsy population". J. Urol., 168: 500, 2002.
18. HERRANZ AMO, F.; VERDÚ TARTAJO, F.; DÍEZ CORDERO, J.Ma . y cols.: "Incidencia de cáncer de próstata en los pacientes sintomáticos con tacto rectal no sospechoso y niveles de PSA mayores de 10 ng/ml". Actas Urol. Esp., 23: 316, 1999.

19. REMZI, M.; DJAVAN, B.; WAMMACK, R. y cols.: "Can total and transition zone volume of the prostate determine whether to perform a repeat biopsy?". Urology, 61: 161, 2003.

*20. DJAVAN, B.; ZLOTTA, A.R.; EKANE, S. y cols.: "Is one set of sextant biopsies enough to rule out prostate cancer? Influence of transition and total prostate volumes on prostate cancer yield". Eur. Urol., 38: 218, 2000.

*21. PRESTI, J.C. Jr.,: "Prostate biopsy: how many cores are enough?”. Urol. Oncol., 21: 135, 2003.

22. KARAKIEWICZ, P.I.; BAZINET, M.; APRIKIAN, A.G.y cols.: "Outcome of sextant biopsy according to gland volume". Urology, 49: 55, 1997.

23. ROEHL, K.A.; ANTENOR, J.A.; CATALONA, W.J.: "Robustness of free prostate specific antigen measurements to reduce unnecessary biopsies in the 2.6 to $4.0 \mathrm{ng} / \mathrm{ml}$ range". J. Urol., 168: 922 , 2002.

**24. CATALONA, W.J.; SOUTHWICK, P.C.; SLAWIN, K.M. y cols.: "Comparison of percent free PSA, PSA density, and age-specific PSA cutoffs for prostate cancer detection and staging". Urology, 56: 255, 2000.

25. RODRÍGUEZ-PATRÓN, R.; MAYAYO DEHESA, T.; BURGOS REVILLA, F.J. y cols.: "Tolerancia y complicaciones de la biopsia transrectal ecodirigida prostática ampliada a diez cilindros. Papel del bloqueo de haces neurovasculares con lidocaina". Arch. Esp. Urol., 58: 989, 2005.

**26. THOMPSON, I.M.; PAULER, D.K.; GOODMAN, P.J. y cols.: "Prevalence of prostate cancer among men with a prostate-specific antigen level $\leq 4.0 \mathrm{ng}$ per mililiter". N. Engl. J. Med., 350: 2239, 2004. 